\title{
Análisis termodinámico del intercambiador de calor de un sistema ORC para el aprovechamiento de calor residual en procesos industriales
}

\section{Thermodynamic analysis of the heat exchanger of an ORC system for the use of residual heat in industrial processes}

\author{
CALDIÑO-HERRERA, Uzziel $\dagger^{*}$, CORNEJO-MONROY, Delfino, TILVALDYEV, Shehret y \\ DÁVALOS-RAMÍREZ, José Omar
}

Universidad Autónoma de Ciudad Juárez, Instituto de Ingeniería y Tecnología

ID $1^{\text {er }}$ Autor: Uzziel, Caldiño-Herrera / ORC ID: 0000-0002-4682-5548, CVU CONACYT ID: 487308

ID $1^{\text {er }}$ Coautor: Delfino, Cornejo-Monroy / ORC ID: 0000-0002-6294-7385, CVU CONACYT ID: 169913

ID $2^{\text {do }}$ Coautor: Shehret, Tilvaldyev / ORC ID: 0000-0003-1671-852X, CVU CONACYT ID: 508563

ID $3{ }^{\text {er }}$ Coautor: José Omar, Dávalos-Ramírez / ORC ID: 0000-0002-6612-5231, CVU CONACYT ID: 336999

DOI: $10.35429 / J M E .2019 .10 .3 .27 .33$

Recibido 28 Abril, 2019; Aceptado 30 Junio, 2019

\section{Resumen}

En este trabajo se presenta la implementación de un sistema basado en ciclo Rankine orgánico acoplado a una descarga de calor de un proceso industrial. El calor desechado se utiliza como fuente energética de entrada en un sistema que utiliza esta energía para evaporar un fluido orgánico y expandirlo en una turbina, en donde se genera potencia mecánica. El sistema consta de 4 procesos y en este trabajo se analiza en particular el intercambiador de calor en donde se transfiere el calor de desecho al fluido orgánico para lograr la evaporación. De acuerdo con la disponibilidad del calor se diseña este dispositivo para lograr el máximo aprovechamiento de la energía. Asimismo, se calcula eficiencia térmica en función de la disponibilidad energética, y se selecciona el mejor fluido de trabajo y las condiciones de operación a través de un análisis termodinámico.

Ciclo Rankine orgánico, Intercambiador de calor, Eficiencia energética

\begin{abstract}
In this paper we present the implementation of a system based on organic Rankine cycle coupled to a heat discharge of an industrial process. Waste heat is used as an energy source input to the system, which uses this energy to evaporate an organic fluid and expand it in a turbine, where mechanical power is produced. The system consists of 4 processes and the heat exchanger is specially analyzed. According to the availability of heat energy, the heat exchanger was designed to achieve the maximum efficiency in the energy system. Likewise, the maximum thermal efficiency of the ORC system is calculated as a function of the available energy, the energy source temperature and the available mass flow rate. By these calculations, the working fluid and the suitable operating conditions were selected through a thermodynamic analysis.
\end{abstract}

Organic Rankine cycle, Heat exchanger, Energetic efficiency

Citación: CALDIÑO-HERRERA, Uzziel, CORNEJO-MONROY, Delfino, TILVALDYEV, Shehret y DÁVALOSRAMÍREZ, José Omar. Análisis termodinámico del intercambiador de calor de un sistema ORC para el aprovechamiento de calor residual en procesos industriales. Revista de Ingeniería Mecánica. 2019. 3-10: 27-33

\footnotetext{
* Correspondencia del Autor (uzziel.caldino@uacj.mx)

$\dagger$ Investigador contribuyendo como primer Autor
} 


\section{Introducción}

El calor residual en procesos es una condición recurrente en la industria global. En general este tipo de calor de desecho presenta condiciones de bajo contenido energético y bajas temperaturas $\left(<100^{\circ} \mathrm{C}\right)$. La implementación del ciclo Rankine orgánico (ORC) en este tipo de fuentes permite aprovechar la energía para producirla en potencia mecánica en un expansor(Frutiger et al., 2016)(Frutiger et al., 2016; Quoilin, Broek, Declaye, Dewallef, \& Lemort, 2013; Quoilin, Declaye, Tchanche, \& Lemort, 2011; Rolónortiz, Acevedo-peñaloza, \& Villamizargonzález, 2019; Yamamoto, Furuhata, Arai, \& Mori, 2001). Al ser pequeña la diferencia de temperaturas bajo la cual opera el sistema basado en ORC, la eficiencia térmica del ciclo es baja, sin embargo, la recuperación de una fracción de la energía que de otra manera sería desechada al ambiente justifica el uso de este tipo de sistemas, sobre todo cuando el calor de desecho se encuentra en flujos másicos continuos. La recuperación de parte de la energía desechada contribuye al aprovechamiento y uso eficiente y sustentable de la energía, permitiendo usar la energía recuperada en sistemas de iluminación o reintegrarla a la red eléctrica.

El ciclo Rankine orgánico consta de 4 procesos termodinámicos, en donde un fluido de trabajo orgánico (base carbono) se evapora a una presión alta, para después ser expandido en una turbina, de donde se extrae potencia mecánica. Debido a la variedad de propiedades termodinámicas y de transporte que existe entre los diferentes fluidos de trabajo posible, una correcta selección de éste debe ser hecha de acuerdo con las condiciones de la fuente energética a aprovechar(Drescher \& Brüggemann, 2007; Hernández-mora et al., 2019; Pethurajan \& Sivan, 2018; Rahbar, Mahmoud, Al-Dadah, Moazami, \& Mirhadizadeh, 2017).

El dispositivo encargado de transferir la energía desde la fuente de calor residual hasta el fluido de trabajo es el intercambiador de calor (evaporador). Por lo tanto, un análisis de termodinámico de este dispositivo se lleva a cabo, partiendo de los datos de un análisis termodinámico del sistema ORC.
Es importante resaltar que el análisis del intercambiador de calor se lleva a cabo una vez que las condiciones del ORC están definidas, cuidando que las temperaturas calculadas en el ORC nunca sobrepasen las que el intercambiador (definido por la fuente energética) permite. Por esta razón, la potencia de la turbina queda definida por la capacidad de la fuente energética, que está ligada con la relación de presiones que ocurre en el ORC. Esto debido a que una de las restricciones del análisis del sistema es que la temperatura de entrada a la turbina corresponde a una condición de sobre calentamiento de $5^{\circ} \mathrm{C}$, y la presión de evaporación es proporcional a la temperatura de evaporación.

Llevando a cabo este análisis, se define el fluido de trabajo que mejor desempeño presente con una fuente energética en particular, y al mismo tiempo arroja una tendencia en el desempeño del sistema con respecto a la relación de presiones.

En este documento se presenta una sección en donde se describe la metodología para llevar a cabo este análisis a través de herramientas computacionales. Además, se presenta una sección con el análisis de resultados y la discusión de éstos, en donde se evalúan diferentes variables dependientes como el flujo másico y la temperatura de la fuente, así como la relación de presión. A continuación, se presentan las conclusiones del trabajo y se finaliza el documento con algunas referencias que ayudaron al desarrollo de este trabajo.

\section{Metodología}

Para llevar a cabo el análisis del intercambiador de calor es necesario definir algunas condiciones del sistema energético, así como las condiciones de la fuente de calor residual. En este trabajo se considera una fuente calor residual como agua líquida a una temperatura $T_{f}$ siempre inferior a $100^{\circ} \mathrm{C}$. Asimismo, se considera una disponibilidad de esta agua a una razón de $\dot{m}_{f}$, cuyo valor máximo se fijó en 1.6 kg/s.

En lo que respecta al sistema ORC, se analizaron 4 fluidos de trabajo: R245fa, R123, $\mathrm{R} 245$ ca y $\mathrm{R} 1233 z d$. La razón de estos fluidos es que su presión de saturación a una temperatura de $25^{\circ} \mathrm{C}$ (que corresponde a la temperatura ambiente) está alrededor de los $101 \mathrm{kPa}$ (presión atmosférica). 
El análisis termodinámico se llevó a cabo utilizado la herramienta CoolProp a través de Python. CoolProp es una base de datos de propiedades termofísicas y de transporte para 123 fluidos. Utiliza ecuaciones de estado de tercer orden $\mathrm{y}$, por lo tanto, como cualquier ecuación de estado, necesita de dos propiedades termodinámicos para calcular cualquier otra propiedad de dicho estado.

Para llevar a cabo el análisis se enumeran los estados termodinámicos del ORC de acuerdo con la Fig. 1, en donde el estado 1 corresponde a la entrada de la bomba y a la salida del condensador. El estado 2 corresponde a la salida de la bomba y a la entrada del evaporador. El estado 3 corresponde a la salida del evaporador y a la entrada de la turbina. Finalmente, el estado 4 corresponde a la salida de la turbina y a la entrada al condensador.

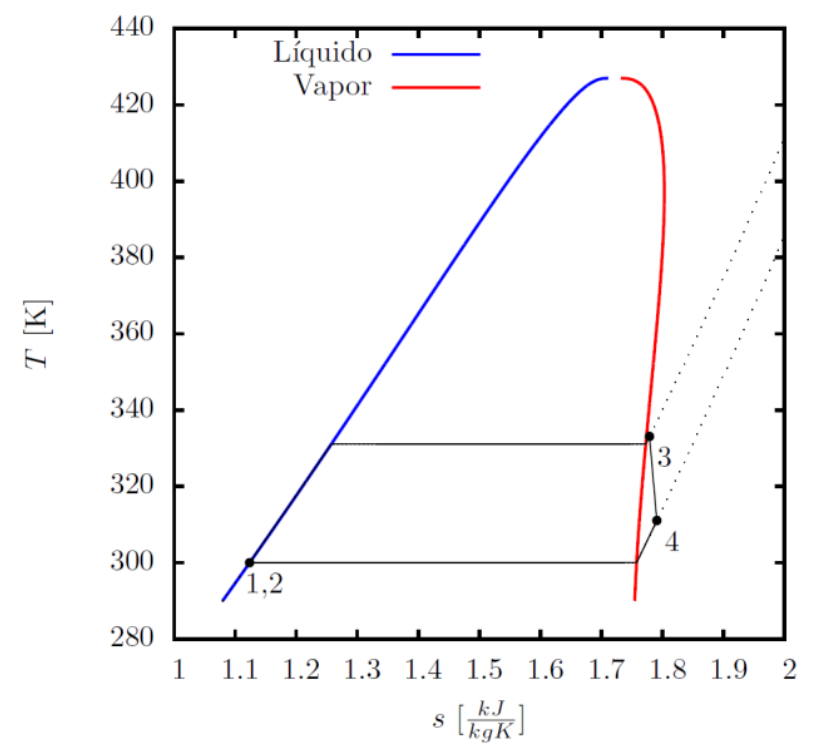

Figura 1 Diagrama Ts de un ciclo ORC mostrando los 4 estados termodinámicos

Siguiendo la numeración de la Fig. 1, en la Tabla 1 se muestra una descripción de los procesos, así como las consideraciones hechas para el análisis termodinámico.

\begin{tabular}{|c|c|}
\hline Proceso & Consideraciones \\
\hline 1-2 (Bomba) & Entrada como líquido saturado \\
\hline $\begin{array}{l}\text { 2-3 } \\
\text { (Evaporador) }\end{array}$ & $\begin{array}{l}\text { Proceso isobárico, condición final } \\
\text { como vapor sobrecalentado } 5^{\circ} \mathrm{C}\end{array}$ \\
\hline 3-4 (Turbina) & $\begin{array}{l}\text { Expansión del fluido considerando } \\
\text { una eficiencia isentrópica de } 75 \%\end{array}$ \\
\hline $\begin{array}{l}\text { 4-1 } \\
\text { (Condensador) }\end{array}$ & $\begin{array}{l}\text { Proceso isobárico, condición final } \\
\text { de líquido saturado }\end{array}$ \\
\hline
\end{tabular}

Tabla 1 Procesos termodinámicos en el ORC y sus consideraciones para el análisis
El evaporador es, como se mencionó anteriormente, el dispositivo en donde interactúa la fuente energética y el sistema ORC. El calor de la fuente es transferido al fluido de trabajo, por lo tanto, para evaluar el evaporador es necesario calcular el desempeño del ORC para posteriormente calcular el flujo másico necesario en el ORC que permita lograr la temperatura de salida del evaporador y por lo tanto, defina la potencia de salida.

El análisis del ORC comienza en la entrada de la bomba, en donde a partir de la condición de saturación y la temperatura de fluido (la cual se establece como $5^{\circ} \mathrm{C}$ por encima de la temperatura ambiente) se calculan el resto de las propiedades termodinámicas $(h, s, \rho, P)$ con CoolProp.

El estado a la salida de la bomba se calcula inicialmente como un proceso isentrópico con una presión igual a la presión de evaporación, la cual se define con la relación de expansión $P_{R}$, de la cual se analiza con valores de $2.5,2.75,3.3 .25$ y 3.5. Posteriormente se calcula la entalpía real considerando una eficiencia en la bomba de 0.8

$h_{2}=h_{1}+\frac{h_{2 s}-h_{1}}{\eta_{b}}$

En donde $h$ es la entalpía, los subíndices 1 y 2 corresponden a la entrada y salida de la bomba respectivamente, el subíndice $s$ es la condición isentrópica y $\eta_{b}$ es la eficiencia de la bomba. El estado 3 se calcula con la presión de evaporación y la temperatura de entrada a la turbina, la cual se define de acuerdo con la Tabla 1 como $5^{\circ} \mathrm{C}$ por encima de la temperatura de saturación.

El estado 4, que corresponde a la salida de la turbina, se calcula inicialmente como un proceso isentrópico con una presión correspondiente a la presión de condensación. Posteriormente se calcula la entalpía real considerando una eficiencia en la turbina de 0.75 .

$h_{4}=h_{3}-\eta_{t}\left(h_{3}-h_{4 s}\right)$

En dónde los subíndices 3 y 4 corresponden a la entrada y salida de la turbina respectivamente, $\eta_{t}$ es la eficiencia de la turbina y el subíndice $s$ es la condición isentrópica de la expansión. 
Una vez que todos los estados están completamente definidos, se calculan los parámetros de desempeño del ORC, los cuales hasta ahora son independientes de la fuente energética, ya que ésta última se calculará para que satisfaga estas condiciones del ORC

$\eta_{O R C}=\frac{\left(h_{3}-h_{4}\right)-\left(h_{2}-h_{1}\right)}{h_{3}-h_{2}}$

El flujo másico del ORC depende de la fuente energética, ya que para lograr alcanzar la temperatura a la salida del evaporador esté se debe ajustar al flujo de calor disponible en el evaporador, el cual está dado por la fuente de calor residual como se muestra en la Ec. (4).

$\dot{m}_{O R C}=\frac{\dot{Q}_{e v a p}}{h_{3}-h_{2}}$

En dónde $\dot{m}_{O R C}$ es el flujo másico del ORC, $\dot{Q}_{\text {evap }}$ es el flujo de calor disponible en el evaporador y que está definido por la fuente de calor residual, y $h_{3}$ y $h_{4}$ son las entalpías de los estados 3 y 4 respectivamente. La potencia entregada por la turbina se calcula de acuerdo con la Ec. (5)

$\dot{W}=\dot{m}_{O R C}\left(h_{3}-h_{4}\right)$

Conociendo las condiciones de operación del ORC, la línea del intercambiador de calor correspondiente a la fuente energética puede ser calculada. Para asegurar un correcto funcionamiento del intercambiador de calor, se debe restringir matemáticamente que la temperatura del agua a la salida del intercambiador sea inferior a la temperatura del fluido de trabajo a la salida de la bomba del ORC.

Esto se hace calculando en primer lugar la entalpía del agua a la salida del intercambiador de calor, y posteriormente, con la presión de trabajo de la fuente energética (considerada en este trabajo como presión atmosférica) se calcula la temperatura correspondiente. Una vez calculada la temperatura se compara con la temperatura a la salida de la bomba de ORC y se descartan todos aquellos casos en los que la temperatura sea menor a $T_{2}+5^{\circ} \mathrm{C}$.

$h_{\text {out }}=h_{\text {in }}-\frac{\dot{Q}_{\text {evap }}}{\dot{m}_{f}}$
En dónde $h_{\text {out }}$ es la entalpía del agua a la salida del intercambiador de calor, $h_{i n}$ es la entalpía de entrada del agua en el intercambiador (la cual corresponde a la temperatura de la fuente) y $\dot{m}_{f}$ es el flujo másico del agua en el evaporador. Este último parámetro es la variable independiente en el análisis del intercambiador, y se calcula la temperatura del agua a la salida del evaporador para diferentes valores de la temperatura de la fuente, siendo este valor siempre inferior a $100^{\circ} \mathrm{C}$. Esto debido a que las fuentes de baja entalpía son aquellas cuya temperatura está por debajo de los $100^{\circ} \mathrm{C}$.

De igual manera, la temperatura de entrada a la turbina se restringe a ser siempre $5^{\circ} \mathrm{C}$ por debajo de la temperatura de la fuente energética.

\section{Resultados y discusión}

Siguiendo la metodología descrita en la sección anterior se calcularon primeramente el desempeño termodinámico del ORC para los 4 fluidos de trabajo y bajo diferentes relaciones de presión $P_{R}$. En las Tablas 2, 3, 4 y 5 se muestran estos resultados para R245fa, R245ca, R123 y R1233zd respectivamente.

Como es de esperarse, al incrementar $P_{R}$ se observa un incremento en la eficiencia térmica y en la potencia producida, así como una disminución en el flujo másico del ORC debido al incremento en la temperatura a la salida del evaporador del fluido de trabajo.

Este comportamiento está presente en los 4 fluidos analizados, sin embargo, la variación de los parámetros para cada fluido es diferente. A continuación, se muestra en análisis de estos resultados para cada fluido de trabajo con incrementos en el valor de $P_{R}$ de 0.5 , iniciando en 2.5 y terminando en 3.5.

\begin{tabular}{|c|c|c|c|c|}
\hline$P_{R}$ & $\begin{array}{c}\eta_{O R C} \\
{[\%]}\end{array}$ & $\begin{array}{l}\dot{m}_{\text {ORC }} \\
{[\mathrm{kg} / \mathrm{s}]}\end{array}$ & $\begin{array}{c}\dot{W}_{\text {turb }} \\
{[\mathbf{k W}]}\end{array}$ & $T_{3}\left[{ }^{\circ} \mathrm{C}\right]$ \\
\hline 2.50 & 5.83 & 0.700 & 8.92 & 63.67 \\
\hline 2.75 & 6.38 & 0.693 & 9.77 & 67.02 \\
\hline 3.00 & 6.87 & 0.686 & 10.53 & 70.15 \\
\hline 3.25 & 7.31 & 0.679 & 11.22 & 73.08 \\
\hline 3.50 & 7.71 & 0.673 & 11.85 & 75.85 \\
\hline
\end{tabular}

Tabla 2 Desempeño del ORC operando con R245fa 
El R245fa presenta de acuerdo con la Tabla 2, incrementos del $0.55 \%, 0.49 \%, 0.44 \%$ y $0.40 \%$ en la eficiencia térmica en cada incremento de $P_{R}$ analizado. La tendencia de una disminución en $\eta_{O R C}$ es muy clara. El incremento total en la eficiencia al pasar de $P_{R}=$ 2.5 a $P_{R}=3.5$ es de $1.88 \%$. La potencia producida tiene una tendencia similar. En términos porcentuales se observan incrementos de $9.52 \%, 7.77 \%, 6.55 \%$, y $5.61 \%$ conforme $P_{R}$ incrementa en los intervalos analizados, logrando un incremento total de $32.84 \%$ en la eficiencia al variar $P_{R}$ de 2.5 a 3.5. El flujo másico del ORC de igual forma disminuye aun que lo hace de manera menos notoria, sin embargo, esto puede influir en el volumen de fluido necesario para operar el ORC.

\begin{tabular}{|c|c|c|c|c|}
\hline$P_{R}$ & $\begin{array}{r}\eta_{\text {ORC }} \\
{[\%]}\end{array}$ & $\begin{array}{c}\dot{m}_{O R C} \\
{[\mathrm{~kg} / \mathrm{s}]}\end{array}$ & $\begin{array}{l}\dot{W}_{\text {turb }} \\
{[\mathrm{kW}]}\end{array}$ & $\begin{array}{c}\boldsymbol{T}_{3} \\
{\left[{ }^{\circ} \mathbf{C}\right]}\end{array}$ \\
\hline 2.50 & 5.63 & 0.656 & 8.56 & 62.36 \\
\hline 2.75 & 6.17 & 0.649 & 9.38 & 65.55 \\
\hline 3.00 & 6.65 & 0.642 & 10.11 & 68.52 \\
\hline 3.25 & 7.08 & 0.636 & 10.78 & 71.32 \\
\hline 3.50 & 7.48 & 0.630 & 11.39 & 73.95 \\
\hline
\end{tabular}

Tabla 3 Desempeño del ORC operando con R245ca

El R245ca presenta de acuerdo con la Tabla 3, incrementos del $0.54 \%, 0.48 \%, 0.43 \%$ y $0.40 \%$ en la eficiencia térmica en cada incremento de $P_{R}$ analizado. La tendencia de una disminución en $\eta_{O R C}$ es muy clara. El incremento total en la eficiencia al pasar de $P_{R}=$ 2.5 a $P_{R}=3.5$ es de $1.85 \%$. La potencia producida tiene una tendencia similar. En términos porcentuales se observan incrementos de $9.57 \%, 7.78 \%, 6.62 \%$, y $5.65 \%$ conforme $P_{R}$ incrementa en los intervalos analizados, logrando un incremento total de $33.06 \%$ en la eficiencia al variar $P_{R}$ de 2.5 a 3.5

\begin{tabular}{|c|c|r|r|r|}
\hline \multicolumn{1}{|c}{$\boldsymbol{P}_{\boldsymbol{R}}$} & $\begin{array}{c}\boldsymbol{\eta}_{\text {ORC }} \\
{[\%]}\end{array}$ & $\begin{array}{c}\dot{\boldsymbol{m}}_{\text {ORC }} \\
{[\mathbf{k g} / \mathbf{s}]}\end{array}$ & \multicolumn{1}{c|}{$\begin{array}{c}\dot{\boldsymbol{W}}_{\text {turb }} \\
{[\mathbf{k W}]}\end{array}$} & $\begin{array}{c}\boldsymbol{T}_{\mathbf{3}} \\
{\left[{ }^{\circ} \mathbf{C}\right]}\end{array}$ \\
\hline $\mathbf{2 . 5 0}$ & 5.92 & 0.790 & 8.99 & 63.52 \\
\hline $\mathbf{2 . 7 5}$ & 6.48 & 0.782 & 9.85 & 66.85 \\
\hline $\mathbf{3 . 0 0}$ & 6.99 & 0.774 & 10.64 & 69.95 \\
\hline $\mathbf{3 . 2 5}$ & 7.45 & 0.768 & 11.34 & 72.87 \\
\hline $\mathbf{3 . 5 0}$ & 7.88 & 0.761 & 11.99 & 75.62 \\
\hline
\end{tabular}

Tabla 4 Desempeño del ORC operando con R123

E1 R123 presenta de acuerdo con la Tabla 4 , incrementos del $0.56 \%, 0.51 \%, 0.46 \%$ y $0.43 \%$ en la eficiencia térmica en cada incremento de $P_{R}$ analizado. La tendencia de una disminución en $\eta_{O R C}$ es muy clara.
El incremento total en la eficiencia al pasar de $P_{R}=2.5$ a $P_{R}=3.5$ es de $1.96 \%$. La potencia producida tiene una tendencia similar. En términos porcentuales se observan incrementos de $9.56 \%, 8.02 \%, 6.57 \%$, y $5.73 \%$ conforme $P_{R}$ incrementa en los intervalos analizados, logrando un incremento total de $33.37 \%$ en la eficiencia al variar $P_{R}$ de 2.5 a 3.5

\begin{tabular}{|c|r|r|r|r|}
\hline $\boldsymbol{P}_{\boldsymbol{R}}$ & \multicolumn{1}{|c|}{$\boldsymbol{\eta}_{\text {ORC }}[\%]$} & $\dot{\boldsymbol{m}}_{\text {ORC }}[\mathrm{kg} / \mathbf{s}]$ & $\dot{\boldsymbol{W}}_{\text {turb }}[\mathrm{kW}]$ & $\boldsymbol{T}_{\mathbf{3}}\left[{ }^{\circ} \mathbf{C}\right]$ \\
\hline $\mathbf{2 . 5 0}$ & 6.05 & 0.704 & 9.25 & 64.62 \\
\hline $\mathbf{2 . 7 5}$ & 6.63 & 0.697 & 10.13 & 68.09 \\
\hline $\mathbf{3 . 0 0}$ & 7.14 & 0.690 & 10.92 & 71.32 \\
\hline $\mathbf{3 . 2 5}$ & 7.60 & 0.684 & 11.64 & 74.36 \\
\hline $\mathbf{3 . 5 0}$ & 8.02 & 0.678 & 12.30 & 77.23 \\
\hline
\end{tabular}

Tabla 5 Desempeño del ORC operando con R1233zd

El R1233zd presenta de acuerdo con la Tabla 5, incrementos del $0.58 \%, 0.51 \%, 0.46 \%$ y $0.42 \%$ en la eficiencia térmica en cada incremento de $P_{R}$ analizado. La tendencia de una disminución en $\eta_{O R C}$ es muy clara. El incremento total en la eficiencia al pasar de $P_{R}=$ 2.5 a $P_{R}=3.5$ es de $1.97 \%$. La potencia producida tiene una tendencia similar. En términos porcentuales se observan incrementos de $9.51 \%, 7.79 \%, 6.59 \%$, y $5.67 \%$ conforme $P_{R}$ incrementa en los intervalos analizados, logrando un incremento total de $32.97 \%$ en la eficiencia al variar $P_{R}$ de 2.5 a 3.5

De estos resultados se observa un mejor comportamiento termodinámico, en términos de eficiencia y potencia producida por parte del R123, seguido del R1233zd. Los refrigerantes R245fa y R245ca muestran comportamientos muy similares. A pesar de que las diferencias son pequeñas, es importante tener en cuenta que al ser sistemas que operan con una diferencia de temperaturas muy baja, un pequeño incremento en potencia producida o en eficiencia térmica resulta en ahorros energéticos, así como en un mejor aprovechamiento energético.

A continuación, se muestran los resultados de evaluar el intercambiador de calor con 3 valores distintos de flujo de calor disponible en la fuente energética: $100 \mathrm{~kW}, 150$ $\mathrm{kW}$ y $200 \mathrm{~kW}$. Recordando que la línea de flujo en el intercambiador de calor que corresponde a la fuente energética, se considera que el fluido de trabajo es agua, se sabe que, independientemente del fluido de trabajo utilizado en el ORC. 
El flujo de calor está definido y la temperatura de salida del agua depende únicamente del flujo másico disponible en la fuente, así como de la temperatura de la fuente energética. En las Figs. 2, 3 y 4 se muestra la variación de la temperatura del agua de calentamiento (proveniente de la fuente energética a aprovechar) a la salida del intercambiador para diferentes valores de temperaturas en la fuente energética.

Sabiendo que existe la restricción de que la temperatura a la salida del evaporador por parte del agua de calentamiento nunca puede ser menor que la temperatura a la salida de la bomba del ORC, existe un flujo másico mínimo que debe lograrse. Este valor depende de la disponibilidad energética en la fuente, la cual es independiente de la temperatura de ésta. Las Figs. 2, 3 y 4 muestran estos datos para valores de flujo de calor disponibles en la fuente energética de $100 \mathrm{~kW}, 150 \mathrm{~kW}$ y $200 \mathrm{~kW}$ respectivamente.

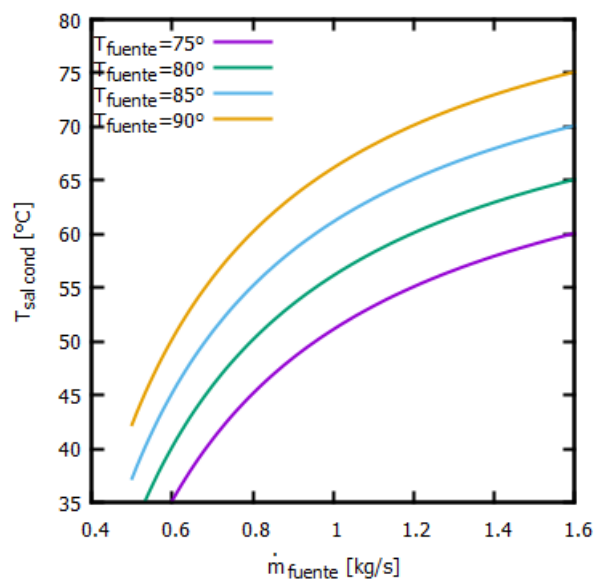

Figura 2 Variación de la temperatura de salida del intercambiador con respecto al flujo másico del mismo para una fuente de calor de $100 \mathrm{~kW}$

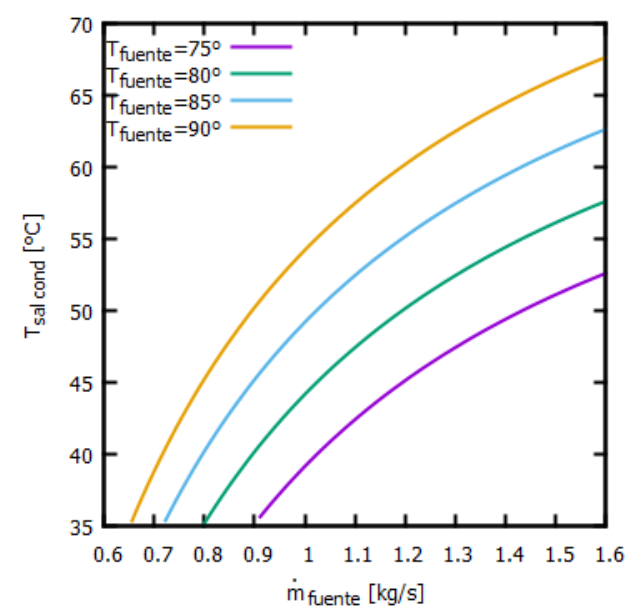

Figura 3 Variación de la temperatura de salida del intercambiador con respecto al flujo másico del mismo para una fuente de calor de $150 \mathrm{~kW}$
Se observa como a mayor disponibilidad energética, el flujo másico mínimo de la fuente puede ser menor, además de que la temperatura de salida del intercambiador de calor disminuye para todas las temperaturas de la fuente. Estas gráficas también muestran que el comportamiento no es lineal, y conforme el flujo másico de la fuente incrementa, la temperatura a la salida del intercambiador tiende a ser la misma para cada caso.

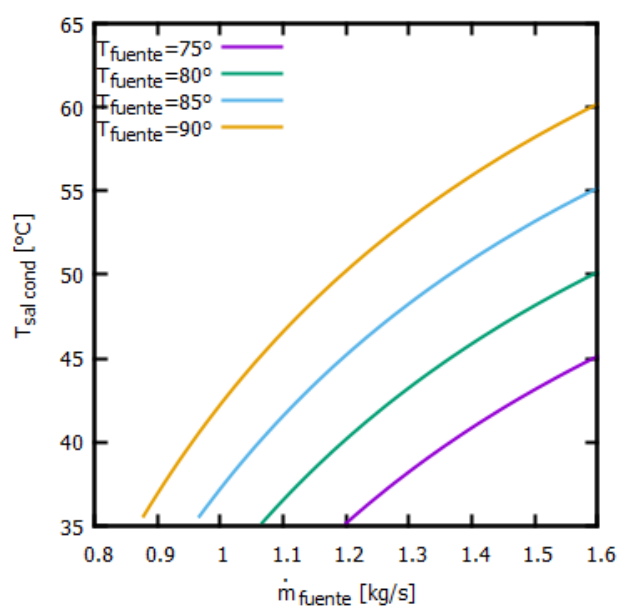

Figura 4 Variación de la temperatura de salida del intercambiador con respecto al flujo másico del mismo para una fuente de calor de $200 \mathrm{~kW}$

\section{Conclusiones}

De acuerdo con lo mostrado en este trabajo se puede concluir lo siguiente:

El análisis termodinámico del intercambiador de calor arroja información sobre la naturaleza de la fuente energética, la cual puede ayudar a redefinir las condiciones de operación o incluso la configuración del ciclo con el fin de lograr un mejor aprovechamiento de la fuente energética de baja entalpía Para una fuente de calor residual con temperatura menor a $90^{\circ} \mathrm{C}$ y un flujo másico disponible menor a $1.6 \mathrm{~kg} / \mathrm{s}$, los fluidos de trabajo R1233zd y R123 resultan ser los que mejor desempeño térmico ofrecen, pudiendo extraer hasta $12 \mathrm{~kW}$ de potencia en la turbina.

- $\quad$ La disponibilidad energética tiene una influencia importante en la operación del intercambiador de calor. Con base en este parámetro se puede elegir un intercambiador con mejor desempeño de acuerdo con el flujo másico mínimo requerido. Esto facilita los cálculos para el diseño de detalle del sistema energético.

CALDIÑO-HERRERA, Uzziel, CORNEJO-MONROY, Delfino, TILVALDYEV, Shehret y DÁVALOS-RAMÍREZ, José Omar. Análisis termodinámico del intercambiador de calor de un sistema ORC para el aprovechamiento de calor residual en procesos industriales. Revista de Ingeniería Mecánica. 2019 


\section{Trabajo futuro}

Se propone emplear un ciclo regenerativo que permita aprovechar la energía en forma de vapor sobrecalentado a la salida de la turbina para precalentar el fluido de trabajo cuando este salga de la bomba. Además, resulta atractivo para un uso sustentable de la energía, utilizar el calor desechado en el condensador para el calentamiento de agua.

\section{Referencias}

Drescher, U., \& Brüggemann, D. (2007). Fluid selection for the Organic Rankine Cycle (ORC) in biomass power and heat plants. Applied Thermal Engineering. https://doi.org/10.1016/j.applthermaleng.2006.0 4.024

Frutiger, J., Andreasen, J., Liu, W., Spliethoff, H., Haglind, F., Abildskov, J., \& Sin, G. (2016). Working fluid selection for organic Rankine cycles - Impact of uncertainty of fluid properties. Energy.

https://doi.org/10.1016/j.energy.2016.05.010

Hernández-mora, I. E., Lugo-leyte, R., Bonillablancas, A. E., López-arenas, T., Salazarpereyra, M., \& Lugo-méndez, H. D. (2019). Exergoeconomic analysis of a compression refrigeration cycle considering the allocation cost of the residue formation process Análisis exergoeconómico de un ciclo de refrigeración por compresión de vapor incluyendo el costo de imputación por la formación del residuo. 86(208), 336-345.

Pethurajan, V., \& Sivan, S. (2018). Experimental Study of an Organic Rankine Cycle Unit Using Dichloromethane as Working Fluid. Inventions, 3(31), 215-217. https://doi.org/10.12691/ajme4-6-2

Quoilin, S., Broek, M. Van Den, Declaye, S., Dewallef, P., \& Lemort, V. (2013). Technoeconomic survey of organic rankine cycle (ORC) systems. Renewable and Sustainable Energy Reviews. https://doi.org/10.1016/j.rser.2013.01.028
Quoilin, S., Declaye, S., Tchanche, B. F., \& Lemort, V. (2011). Thermo-economic optimization of waste heat recovery Organic Rankine Cycles. Applied Thermal Engineering. https://doi.org/10.1016/j.applthermaleng.2011.0 5.014

Rahbar, K., Mahmoud, S., Al-Dadah, R. K., Moazami, N., \& Mirhadizadeh, S. A. (2017). Review of organic Rankine cycle for small-scale applications. Energy Conversion and Management.

https://doi.org/10.1016/j.enconman.2016.12.02 3

Rolón-ortiz, H., Acevedo-peñaloza, C., \& Villamizar-gonzález, Y. (2019). Análisis térmico , modelamiento matemático y simulación de un reactor de agitación discontinuo para volumen específico Thermal analysis , mathematical modeling and simulation of a discontinuous agitation reactor for specific volume. 18(1), 39-48.

Yamamoto, T., Furuhata, T., Arai, N., \& Mori, K. (2001). Design and testing of the organic rankine cycle. Energy. https://doi.org/10.1016/S0360-5442(00)000633 